\title{
APPROXIMATION BY BOUNDED ANALYTIC FUNCTIONS: UNIFORM CONVERGENCE AS IMPLIED BY MEAN CONVERGENCE $\left({ }^{1}\right)$
}

\author{
BY \\ J. L. WALSH
}

In three recent notes [1], [2], [3] I have discussed uniform convergence by polynomials (in the complex variable) to a given function as a consequence of convergence in the mean of those polynomials to the given function, and also convergence in the mean of one order as a consequence of convergence in the mean of a lower order. The present note contains analogs of those results, but now for approximation by bounded analytic functions. As a first illustration of the new results, we have

THEOREM 1. Let $\Gamma$ be an analytic Jordan curve contained in the simply-connected region $D$ of the z-plane, and suppose we have for some function $f(z)$ continuous on $\Gamma$ and functions $f_{n}(z)$ analytic in $D$

$$
\begin{aligned}
& \int_{\Gamma}\left|f(z)-f_{n}(z)\right|^{q}|d z| \leqq A / n^{q \alpha}, \quad q>0, \\
& \left|f_{n}(z)\right| \leqq A R^{n}, \quad z \text { in } D .
\end{aligned}
$$

Then for $\alpha+1 / p-1 / q>0$ and $0<q<p \leqq \infty$ we have for the pth power norm on $\Gamma$

$$
\left\|f(z)-f_{n}(z)\right\|_{p} \leqq A / n^{\alpha+(1 / p)-(1 / q)} .
$$

Here and below the constants $A$ are independent of $n$ and $z$, and may change from one inequality to another.

For $p=\infty$ we consider the first member of (3) as the Tchebycheff (uniform) norm of $\left[f(z)-f_{n}(z)\right]$ on $\Gamma$, with a similar interpretation in later formulas. As is usual in the study of convergence by bounded analytic functions, we note (see for instance [4, §2.2]) that there exist for each $n$ and $N$ polynomials $P_{n, N}(z)$ of respective degrees $N$ such that we have

$$
\left|f_{n}(z)-p_{n, N}(z)\right| \leqq A R^{n} / R_{1}^{N}, \quad z \text { on } \Gamma, \quad R_{1}>1 .
$$

If we choose the integer $\lambda$ so large that $R_{1}^{\lambda}>R$, there follow

$$
\begin{aligned}
\left|f_{n}(z)-p_{n, \lambda n}(z)\right| & \leqq A\left(R / R_{1}^{\lambda}\right)^{n}, \quad z \text { on } \Gamma, \\
\int_{\Gamma}\left|f_{n}(z)-p_{n, \lambda n}(z)\right|^{q}|d z| & \leqq A / n^{q \alpha} .
\end{aligned}
$$

Presented to the Society, August 23, 1966; received by the editors September 9, 1966.

( $\left.{ }^{1}\right)$ Research sponsored (in part) by the Air Force Office of Scientific Research. 
Standard algebraic inequalities depending on $q$ yield by (1) and (6)

$$
\int_{\Gamma}\left|f(z)-p_{n, \lambda n}(z)\right|^{q}|d z| \leqq A_{0} / n^{q \alpha} .
$$

The polynomials $p_{n, \lambda n}(z)$ are defined only for the degrees $\lambda n=\lambda, 2 \lambda, 3 \lambda, \ldots$, but to obtain polynomials $P_{m}(z)$ for all degrees we may set $P_{m}(z)=p_{n, \lambda n}(z)$ for $\lambda n \leqq m<\lambda(n+1)$, whence for $m=1,2,3, \ldots$

$$
\int_{\Gamma}\left|f(z)-P_{m}(z)\right|^{q}|d z| \leqq \frac{A_{0}}{n^{q \alpha}} \leqq \frac{A_{1}}{[\lambda(n+1)]^{q \alpha}} \leqq \frac{A_{1}}{m^{q \alpha}},
$$

provided $A_{1} \geqq A_{0} \lambda^{q \alpha}(n+1)^{q \alpha} / n^{q \alpha}$ for all $n$. Consequently $f(z)$ has various known properties on $\Gamma$. Thus by [3, Theorem 11] we have (since $\alpha+1 / p>1 / q$ )

$$
\left\|f(z)-p_{n, \lambda n}(z)\right\|_{p} \leqq A / n^{\alpha+(1 / p)-(1 / q)} .
$$

Inequality (8) together with (5) now yields (3).

The reader may notice the validity of

COROLlary 1. In Theorem 1 the second member of (1) may be replaced by A $\varepsilon_{n}^{q}$, where $\varepsilon_{n}(>0)$ is monotonic nonincreasing as $n$ increases, is such that $r^{n}=o\left(\varepsilon_{n}\right)$ for every $r(<1)$, with the property $\varepsilon_{n}=O\left(\varepsilon_{\lambda n}\right)$ whenever integral $\lambda>1$, and where the expression $\left(2^{m-1} \leqq n<2^{m}\right)$,

$$
\begin{gathered}
\frac{2^{m r} \varepsilon_{n}+2^{(m+1) r} \varepsilon_{2^{m}}+2^{(m+2) r} \varepsilon_{2^{m+1}}+\cdots, \quad}{n^{r} \varepsilon_{n}}, \quad p \geqq 1, \\
\frac{\left(2^{m}\right)^{p r} \varepsilon_{n}^{p}+\left(2^{m+1}\right)^{p r} \varepsilon_{2^{m}}^{p}+\left(2^{m+2}\right)^{p r} \varepsilon_{2^{p}+1}^{p}+\cdots}{n^{p r} \varepsilon_{n}^{p}}, \quad p<1,
\end{gathered}
$$

where $r=1 / q-1 / p$, has a meaning and is bounded as $n \rightarrow \infty$; the second member of (3) is to be replaced by $A n^{1 / q-1 / p} \varepsilon_{n}$, assumed to approach zero.

In the proof, the second members of (6), (7), and (8) are to be replaced by $A \varepsilon_{n}^{q}$, $A_{0} \varepsilon_{n}^{q}, A n^{1 / q-1 / p} \varepsilon_{n}$ respectively.

Both Theorem 1 and Corollary 1 can be modified in hypothesis and conclusion so that the first member of (1) is a double integral taken over the interior of $\Gamma$, as we now indicate.

First we state a result [3, Theorem 14] on degree of convergence by polynomials:

THFOREM 2. Let $E$ be the closed interior of an analytic Jordan curve, and let a function $f(z)$ continuous on $E$ and polynomials $p_{n}(z)$ of respective degrees $n$ be given such that we have for the qth power norm on $E$

$$
\left\|f(z)-p_{n}(z)\right\|_{q}^{\prime} \leqq \varepsilon_{n}, \quad q>0
$$

and where $\varepsilon_{n}$ has the first three properties of Corollary 1. Let us suppose the expression (9) or (10) with $r$ replaced by $s=2 / q-2 / p$ exists and is bounded as $n \rightarrow \infty$, where $2^{m-1} \leqq n<2^{m}$. Then we have for $0<q<p \leqq \infty$,

$$
\left\|f(z)-p_{n}(z)\right\|_{p}^{\prime} \leqq A n^{2 / q-2 / p} \varepsilon_{n},
$$


where the second member is supposed to approach zero. In particular we may choose $\varepsilon_{n}=n^{-\alpha}, \alpha>2 / q-2 / p$.

Second, we indicate the analog of Theorem 2 for approximation by bounded analytic functions, which is thus an extension of Theorem 2 , in the spirit of Theorem 1 and its Corollary as an extension of [1, Theorem 2].

THEOREM 3. Let $E$ be the closed interior of an analytic Jordan curve contained in the simply-connected region $D$, and suppose some function $f(z)$ analytic interior to $E$, continuous on $E$, and functions $f_{n}(z)$ analytic throughout $D$ satisfy

$$
\begin{aligned}
\iint_{E}\left|f(z)-f_{n}(z)\right|^{q} d S & \leqq A / n^{q \alpha}, & & q>0 \\
\left|f_{n}(z)\right| & \leqq A R^{n}, & & z \text { in } D .
\end{aligned}
$$

Then for $\alpha>2 / q-2 / p$ and $0<q<p \leqq \infty$ we have

$$
\left\|f(z)-f_{n}(z)\right\|_{p}^{\prime} \leqq A / n^{\alpha+2 / p-2 / q} .
$$

Theorem 3 follows by the methods of proof of [1, Theorem 4] and the present Theorem 2. Like Theorem 1, Theorem 3 can be generalized in a suitable corollary:

COROLlARY 1. In Theorem 3 the second member of (13) may be replaced by $A \varepsilon_{n}^{q}$ where $\varepsilon_{n}(>0)$ is arbitrary monotonic nonincreasing, and is such that $r^{n}=o\left(\varepsilon_{n}\right)$ for every $r(<1)$, with the property $\varepsilon_{n}=O\left(\varepsilon_{\lambda n}\right)$ whenever integral $\lambda>1$, and where the expression (9) or (10) with $r$ replaced by $s$ has a meaning and is bounded as $n \rightarrow \infty$, with $2^{m-1} \leqq n<2^{m}$. The second member of (15) is to be replaced by $A n^{2 / q-2 / p} \varepsilon_{n}$, and is assumed to approach zero.

The preceding results, primarily relating to approximation by bounded analytic functions, have an analog for approximation on a curve rather than in a region:

TheOREM 4. Let $\Gamma$ be an analytic Jordan curve contained in a region $D$ not necessarily simply-connected, and suppose we have for some function $f(z)$ continuous on $\Gamma$ and functions $f_{n}(z)$ analytic in $D$

$$
\begin{aligned}
\int_{\Gamma}\left|f(z)-f_{n}(z)\right|^{q}|d z| & \leqq A \varepsilon_{n}^{q}, & & q>0, \\
\left|f_{n}(z)\right| & \leqq A R^{n}, & & z \text { in } D,
\end{aligned}
$$

where $\varepsilon_{n}(>0)$ is monotonic nonincreasing, is such that $r^{n}=o\left(\varepsilon_{n}\right)$ for every $r(<1)$, and with the property $\varepsilon_{n}=O\left(\varepsilon_{\lambda n}\right)$ whenever integral $\lambda>1$, and where the expression (9) or (10) has a meaning and is bounded as $n \rightarrow \infty$, with $2^{m-1} \leqq n<2^{m}$. Then if $n^{1 / q-1 / p} \varepsilon_{n} \rightarrow 0$ and $0<q<p \leqq \infty$, we have for the pth power norm on $\Gamma$

$$
\left\|f(z)-f_{n}(z)\right\|_{p} \leqq A n^{1 / q-1 / p} \varepsilon_{n} .
$$

In particular we may choose $\varepsilon_{n}=n^{-\alpha}, \alpha>1 / q-1 / p$. 
In the proof of Theorem 4, we assume the origin to lie interior to $\Gamma$, approximate the $f_{n}(z)$ on $\Gamma$ by polynomials in $z$ and $1 / z$, and use the method of [3]. Details are left to the reader.

Theorem 4 applies to approximation on the unit circumference $\Gamma$ to a real or complex function $f(z)$ by real or complex bounded analytic functions $f_{n}(z)$, or with the substitution $z=e^{i \theta}$, approximation on the real line $-\infty<\theta<\infty$ to a function with period $2 \pi$ by bounded analytic functions with period $2 \pi$ in a strip containing the line. In particular if $f_{n}(z)$ is a polynomial in $z$ and $1 / z$ of degree $n$ satisfying (16), then (17) follows if $D$ is an annulus containing $\Gamma$ in its interior with boundary components having 0 as center, and $f_{n}\left(e^{i \theta}\right)$ is a trigonometric polynomial of order $n$. Compare here [2, Theorems 6-9].

Theorem 4 suggests approximation by bounded analytic functions in a multiply connected region, as measured by a line integral over the boundary:

THEOREM 5. Let $E$ be a closed bounded region whose boundary $\Gamma$ consists of a finite number of mutually disjoint analytic Jordan curves, and which lies in a region $D$. Suppose for some function $f(z)$ analytic interior to $E$ and continuous on $E$ and for functions $f_{n}(z)$ analytic in $D$ we have (16) and (17), where $\varepsilon_{n}$ satisfies the conditions of Theorem 4. Then if $n^{1 / q-1 / p} \varepsilon_{n} \rightarrow 0$ and $0<q<p \leqq \infty$ we have (18) for the pth power norm on $\Gamma$. In particular we may choose $\varepsilon_{n}=n^{-\alpha}, \alpha>1 / q-1 / p$.

To prove Theorem 5, we merely apply Theorem 4 to each component of $\Gamma$ and of $f(z)$.

Our primary topic in the foregoing theorems is degree of uniform convergence of the $f_{n}(z)$ to $f(z)$, so it is natural to assume those functions continuous in the closed regions considered. Some comments on uniform convergence in subregions as a consequence of mean convergence on the boundary or over a region are made in $[5, \S 5.8]$.

We proceed to study the analog of Theorem 5, using as norm a double integral, whose proof is more involved than that of Theorem 5:

THEOREM 6. Let $E$ be a closed bounded region whose boundary $\Gamma$ consists of a finite number of mutually disjoint analytic Jordan curves, and which lies in a region $D$. Suppose for some function $f(z)$ analytic interior to $E$, continuous on $E$, and for functions $f_{n}(z)$ analytic in $D$ we have

$$
\iint_{E}\left|f(z)-f_{n}(z)\right|^{q} d S \leqq A \varepsilon_{n}^{q}, \quad q>0,
$$

and (14), where $\varepsilon_{n}$ satisfies the conditions of Corollary 1 to Theorem 3. Then if $n^{2 / q-2 / p} \varepsilon_{n} \rightarrow 0$ and $0<q<p \leqq \infty$ we have

$$
\left\|f(z)-f_{n}(z)\right\|_{p}^{\prime} \leqq A n^{2 / q-2 / p} \varepsilon_{n},
$$

where we assume the second member approaches zero. In particular we may choose $\varepsilon_{n}=n^{-\alpha}, \alpha>2 / q-2 / p$. 
Let the components of $\Gamma$ be $\Gamma_{1}, \Gamma_{2}, \ldots, \Gamma_{v}$ where $\Gamma_{1}$ bounds a closed finite region $E_{1}$ containing $E$, and $\Gamma_{j}(j>1)$ bounds a closed infinite region $E_{j}$ containing $E$. Let $\Gamma_{j}^{\prime}$ be a variable analytic Jordan curve interior to $E(j=1,2, \ldots, \nu)$ which together with $\Gamma_{j}$ bounds a closed annular region $G_{j}$, where the $G_{j}$ are mutually disjoint. Since the curve $\Gamma_{j}^{\prime}$ lies in $E$, there follows from (19) by [5, §5.3, Lemma 2]

$$
\left|f(z)-f_{n}(z)\right| \leqq A \varepsilon_{n}, \quad z \text { on } \Gamma_{j}^{\prime}
$$

where $A$ varies with $\Gamma_{j}^{\prime}$.

If $z$ is an arbitrary point interior to $E$, the $\Gamma_{j}^{\prime}$ can be chosen so that $z$ lies exterior to the $G_{j}$, and indeed $z$ lies interior to the region bounded by all $\nu$ of the $\Gamma_{j}^{\prime}$. For this point $z$, the value of $f(z)$ is represented by the Cauchy integral of $f(z)$ over $\sum \Gamma_{j}^{\prime}$, so we may write $f(z)=\sum f^{(j)}(z)$ for $z$ interior to $E$, and similarly $f_{n}(z)$ $\equiv \sum f_{n}^{(j)}(z)$ for $z$ interior to $E$, where the $\nu$ components $f^{(j)}(z)$ and $f_{n}^{(j)}(z)$ of $f(z)$ and $f_{n}(z)$ are represented by the Cauchy integrals of $f(z)$ and $f_{n}(z)$ over the respective $\Gamma_{j}^{\prime}$ but are independent of the $\Gamma_{j}^{\prime}$ having the required properties. By inequality (21) we have for $z$ on any closed subset of $E$ disjoint from $G_{j}$

$$
\left|f^{(j)}(z)-f_{n}^{(j)}(z)\right| \leqq A \varepsilon_{n} \quad(j=1,2, \ldots, \nu) .
$$

The functions $f^{(j)}(z)$ and $f_{n}^{(j)}(z)$ are defined throughout the interior of $E_{j}$ and inequality (22) is valid also for $z$ on $E_{j}-G_{j}$ minus a neighborhood of $\Gamma_{j}^{\prime}$.

It is natural to attempt to use (22) to obtain an inequality on the functions $f^{(j)}(z)-f_{n}^{(j)}(z)$ on each $E_{k}$, but this procedure is complicated by the fact that $\nu-1$ of these regions are infinite and the surface integral norm cannot be used directly.

We may choose points $\alpha_{1}=\infty, \alpha_{2}, \ldots, \alpha_{v}$ fixed in the respective regions $D_{1}, D_{2}, \ldots, D_{v}$ exterior to $E$ bounded by $\Gamma_{1}, \Gamma_{2}, \ldots, \Gamma_{v}$, and choose in each $D_{j}$ and in $D$ an analytic Jordan curve $\Gamma_{j}^{\prime \prime}$ separating $\alpha_{j}$ from $E$ but so that the region $D_{0}$ bounded by $\sum \Gamma_{j}^{\prime \prime}$ contains no point not in $D$. The components of $f_{n}(z)$ already defined can be represented by Cauchy integrals of $f_{n}(z)$ over the curves $\Gamma_{j}^{\prime \prime}$, and we have by (14)

$$
\left|f_{n}^{(j)}(z)\right| \leqq A R^{n}, \quad z \text { in } D_{j}^{0},
$$

where $D_{j}^{0}$ is a suitable closed region containing $E_{j}$ in its interior and separated by $\Gamma_{j}^{\prime \prime}$ from $\alpha_{j}$.

We fasten our attention now on $E_{1}, f^{(1)}(z)$, and $f_{n}^{(1)}(z)$. Inequality (22) yields

$$
\begin{aligned}
\left|f^{(j)}(z)-f_{n}^{(j)}(z)\right| & \leqq A \varepsilon_{n}, \quad z \text { on } G_{1}, \quad j>1, \\
\iint_{G_{1}} \sum_{j>1}\left|f^{(j)}(z)-f_{n}^{(j)}(z)\right|^{q} d S & \leqq A \varepsilon_{n}^{q},
\end{aligned}
$$

and by (19) with the integral over $G_{1}$ there follows

$$
\iint_{G_{1}}\left|f^{(1)}(z)-f_{n}^{(1)}(z)\right|^{q} d S \leqq A \varepsilon_{n}^{q} .
$$


The point set $G_{1}$ is to some extent variable, so we deduce also by (22) and by the finiteness of the area of $E_{1}$,

$$
\iint_{E_{1}-G_{1}}\left|f^{(1)}(z)-f_{n}^{(1)}(z)\right|^{q} d S \leqq A \varepsilon_{n}^{q},
$$

where the new $E_{1}-G_{1}$ contains in its interior the partial boundary $\Gamma_{1}^{\prime}$ of the $G_{1}$ in (24). Then by (24) and (25) we have

$$
\iint_{E_{1}}\left|f^{(1)}(z)-f_{n}^{(1)}(z)\right|^{q} d S \leqq A \varepsilon_{n}^{q}
$$

By (23) and (26) we are in a position to apply Corollary 1 to Theorem 3, which establishes

$$
\iint_{E}\left|f^{(1)}(z)-f_{n}^{(1)}(z)\right|^{p} d S \leqq A n^{2 p / q-2} \varepsilon_{n}^{p}
$$

the integral may be taken over $E_{1}$ or $E$. This proof does not apply directly to (27) with 1 replaced by $j(>1)$ because the area of $E_{j}$ is then infinite.

However, for $j>1$ we make a linear transformation $w=\phi(z)$ that carries $\alpha_{j}$ to infinity, which then transforms $E_{j}$ into a finite region of the $w$-plane. By the method of proof of (24) we establish

$$
\iint_{G_{j}}\left|f^{(j)}(z)-f_{n}^{(j)}(z)\right|^{q} d S \leqq A \varepsilon_{n}^{q}, \quad d S=d S_{z} .
$$

With the transformation $w=\phi(z), z=\psi(w)$, we may set $d S_{w}=\left|\phi^{\prime}(z)\right|^{2} d S_{z}$, where $\left|\phi^{\prime}(z)\right|$ is bounded and bounded from zero except near $z=\alpha_{j}$ and $z=\infty$ and their images, whence for the integral over the image of $G_{j}$

$$
\iint\left|f^{(j)}[\psi(w)]-f_{n}^{(j)}[\psi(w)]\right|^{q} d S_{w} \leqq A \varepsilon_{n}^{q} .
$$

By (22) we may write (28) for the integral over the image of a new $E_{j}-G_{j}$ containing the partial boundary $\Gamma_{j}^{\prime}$ of the previously used $G_{j}$ (by the boundedness of the area of the image of $E_{j}$ ). There follows for the integral over the image of $E_{j}$ this same inequality (28).

By virtue of (23) interpreted in the $w$-plane, we can now apply Corollary 1 to Theorem 3, which proves for the integral over the image of $E_{j}$ or $E$

$$
\iint\left|f^{(j)}[\psi(w)]-f_{n}^{(j)}[\psi(w)]\right|^{p} d S_{w} \leqq A n^{2 p / q-2} \varepsilon_{n}^{p} .
$$

We use this integral over the image of $E$, on which $\psi^{\prime}(w)$ is bounded and bounded from zero, so there follows $(j>1)$

$$
\iint_{E}\left|f^{(j)}(z)-f_{n}^{(j)}(z)\right|^{p} d S_{z} \leqq A n^{2 p / q-2} \varepsilon_{n}^{p},
$$

and (27) yields (20), which completes the proof of Theorem 6. 
We add now some general comments on the theorems already proved. If the $f_{n}(z)$ of Theorem 1 are polynomials of respective degrees $n$ satisfying (1), inequality (2) is a consequence of (1). For inequality (1) implies the boundedness $(n \rightarrow \infty)$ of

$$
\int_{\Gamma}\left|f_{n}(z)\right|^{q}|d z|
$$

and (2) follows where $D$ is an arbitrary finite region bounded by a level locus $\Gamma_{R}$, by $\left[5, \S 5.2\right.$, Lemma]. Here we denote generically by $\Gamma_{\rho}(\rho>1)$ the locus $|\phi(z)|=\rho$ in the complement $K$ of $E$, where $w=\phi(z)$ maps $K$ onto $|w|>1, \phi(\infty)=\infty$. A more general remark can be made:

REMARK. Let $E$ be a closed limited point set whose complement is simply connected and whose boundary $\Gamma$ has positive linear measure. If the rational functions $f_{n}(z)$ of respective degrees $n$ satisfy (1), and if the poles of the $f_{n}(z)$ have no limit point on $E$, then for a suitably chosen region $D$ containing $E$, inequality (2) is satisfied.

An inequality

$$
\int_{\Gamma}\left|f_{n}(z)\right|^{q}|d z| \leqq L^{q}, \quad q>0,
$$

follows by the method of treatment of (29). If the $f_{n}(z)$ have no poles on or interior to $\Gamma_{B}, B>1$, then $\left[5, \S 9.8\right.$, Lemma III] we have for $z$ on and within $\Gamma_{z}$

$$
\left|f_{n}(z)\right| \leqq A L[(B Z-1) /(B-Z)]^{n}, \quad 1<Z<B,
$$

so we may choose $D$ as the closed interior of $\Gamma_{z}$ by identifying (31) with (2).

The Remark just established deserves a number of additional comments.

$1^{\circ}$. It is immaterial whether the hypothesis of the Remark is chosen as (1) or as the replacement of (1) as in Corollary 1 to Theorem 1. In either case we obtain (30) at once.

$2^{\circ}$. Let the hypothesis (1) of the Remark be replaced by the inequality

$$
\left|f(z)-f_{n}(z)\right| \leqq A \varepsilon_{n}, \quad z \text { on } \Gamma \text {. }
$$

The uniform boundedness of the rational functions $f_{n}(z)$ follows on $\Gamma$, and an appropriate lemma $\left[5, \S 9.7\right.$, Lemma I] yields (31) for $z$ on or within $\Gamma_{z}$ if all poles of the $f_{n}(z)$ lie exterior to $\Gamma_{B}, 1<Z<B$. Thus $D$ can be chosen as the interior of $\Gamma_{z}$. This comment is of interest in connection with approximation also in the real domain, as in [6].

$3^{\circ}$. The hypothesis of the Remark may be replaced by an inequality for the double integral:

$$
\iint_{E}\left|f(z)-f_{n}(z)\right|^{q} d S \leqq A \varepsilon_{n}^{q}
$$

say under the hypothesis of Theorem 2, where the rational functions $f_{n}(z)$ of respective degrees $n$ have no limit point of poles on $E$. We obtain the boundedness of the integrals

$$
\iint_{E}\left|f_{n}(z)\right|^{q} d S
$$


hence $\left[5, \S 5.3\right.$, Lemma II] there follows on an arbitrary closed region $E^{\prime}$ interior to $E$ the uniform boundedness of the $f_{n}(z)$. Let the poles of the $f_{n}(z)$ have no limit point on or exterior to $E_{\rho}, \rho>1$. Then for $E^{\prime}$ sufficiently large in $E$, the locus $\left(E^{\prime}\right)_{\rho}$ can be chosen as near $E_{\rho}$ as desired (but interior to $E_{\rho}$ ), so in particular we can choose $E^{\prime}$ so that $\left(E^{\prime}\right)_{\rho}$ contains in its interior some $E_{B}, B>1$, which contains $E$ in its interior. If $\left|f_{n}(z)\right| \leqq L$ for $z$ on $E^{\prime}$, we have for $z$ on $\left(E^{\prime}\right)_{z}$ (chosen to contain $E$ and be contained in $E_{B}$ )

$$
\left|f_{n}(z)\right| \leqq A L[(\rho Z-1) /(\rho-Z)]^{n}, \quad 1<Z<\rho,
$$

by $\left[5, \S 9.7\right.$, Lemma I]. The region $D$ can be chosen as the interior of $\left(E^{\prime}\right)_{z}$.

$4^{\circ}$. The Remark can be extended so as to apply even if the complement of $E$ is not simply connected, provided the boundary of $E$ consists of a finite number of mutually disjoint analytic Jordan curves. We assume that $f_{n}(z)$ is a sequence of rational functions of respective degrees $n$ whose poles have no limit point on $E$; it follows for instance that inequality (16) implies (17). Compare here Theorem 4 and $[2$, Theorems 6,7 , and 8$]$.

$5^{\circ}$. The reasoning involved in the Remark may apply even if the approximating functions $f_{n}(z)$ are no longer rational functions, provided each $f_{n}(z)$ is meromorphic with not more than $n$ poles in each of one or more suitable regions. For instance we might consider approximation on a Jordan curve $E$ containing in its interior a closed simply connected region $E_{0}$, where the functions $f_{n}(z)$ are respectively meromorphic with no more than $n$ poles in the complement $E_{1}$ of $E_{0}$, continuous and bounded on the boundary of $E_{0}$.

Throughout this paper we have assumed for simplicity that the Jordan curves involved are analytic. That assumption can be somewhat weakened, as by the use of curves of type $B$ in [1], and of type $B^{\prime}$ in [2].

\section{REFERENCES}

1. J. L. Walsh, Approximation by polynomials: uniform convergence as implied by mean convergence, Proc. Nat. Acad. Sci. U.S.A. 55 (1966), $20-25$.

2. - Approximation by polynomials: uniform convergence as implied by mean convergence. II, Proc. Nat. Acad. Sci. U.S.A. 55 (1966), 1405-1407.

3. - Approximation by polynomials: uniform convergence as implied by mean convergence. III, Proc. Nat. Acad. Sci. U.S.A. 56 (1966), 1406-1408.

4. - - Approximation by bounded analytic functions, Mémor. Sci. Math., Fasc. 144, Gauthier-Villars, Paris, 1960.

5. - Interpolation and approximation, Colloq. Publ., Vol. 20, Amer. Math. Soc., Providence, R. I., 1935.

6. - Degree of approximation by rational functions and polynomials, Michigan Math. J. (to appear).

UNIVERSITY OF MARYLAND, College Park, Maryland 